\title{
OPEN Acute hyperoxia reveals tonic influence of peripheral chemoreceptors on systemic vascular resistance in heart failure patients
}

\author{
Stanislaw Tubek ${ }^{1,2 \bowtie}$, Piotr Niewinski ${ }^{1,2}$, Bartlomiej Paleczny ${ }^{3}$, Anna Langner-Hetmanczuk ${ }^{1,2}$,
} Waldemar Banasiak ${ }^{4}$ \& Piotr Ponikowski ${ }^{1,2}$

Peripheral chemoreceptors' (PCh) hyperactivity increases sympathetic tone. An augmented acute ventilatory response to hypoxia, being a marker of $\mathrm{PCh}$ oversensitivity, was also identified as a marker of poor prognosis in HF. However, not much is known about the tonic (chronic) influence of PCh on cardio-respiratory parameters. In our study $30 \mathrm{HF}$ patients and 30 healthy individuals were exposed to $100 \%$ oxygen for $1 \mathrm{~min}$ during which minute ventilation and hemodynamic parameters were noninvasively recorded. Systemic vascular resistance (SVR) and mean arterial pressure (MAP) responses to acute hyperoxia differed substantially between $\mathrm{HF}$ and control. In HF hyperoxia caused a significant drop in SVR in early stages with subsequent normalization, while increase in SVR was observed in controls. MAP increased in controls, but remained unchanged in HF. Bilateral carotid bodies excision performed in two HF subjects changed the response to hyperoxia towards the course seen in healthy individuals. These differences may be explained by the domination of early vascular reaction to hyperoxia in $\mathrm{HF}$ by vasodilation due to the inhibition of augmented tonic activity of PCh. Otherwise, in healthy subjects the vasoconstrictive action of oxygen remains unopposed. The magnitude of SVR change during acute hyperoxia may be used as a novel method for tonic PCh activity assessment.

Myocardial injury causing heart failure with reduced ejection fraction (HFrEF) is a beginning of autonomic storm, which leads to further disease progression affecting both morbidity and mortality ${ }^{1}$. Numerous pathophysiological mechanisms, shifting the autonomic balance towards sympathetic domination, have been described including: decreased input from arterial baroceptors and pulmonary stretch receptors and increased signaling from renal afferents, muscle ergoreceptors, cardiac receptors and chemoreceptors ${ }^{1}$. Despite constant improvement in pharmacological and device therapy life expectancy in HFrEF is still poor with the 1-year all-cause mortality rate at $23.6 \%$ for acute cases and $6.4 \%$ for chronic ones ${ }^{2}$. Hence, the implementation of novel approaches, engaging not-yet-covered mechanisms, is necessary to improve patients' survival.

One of the mentioned pathophysiological reflexes leading to HFrEF progression is an activation of peripheral chemoreceptors (PCh). In experimental animal models deterioration in left ventricular ejection fraction (LVEF) was clearly accompanied by an increase in peripheral chemoreceptors (PCh) sensitivity and sympathetic overactivity $^{3,4}$. Bilateral PCh deactivation in the same models improved autonomic balance, decreased arrhythmic burden, and restrained left ventricle wall fibrosis preventing further decline in LVEF when compared to sham animals ${ }^{3,4}$. Moreover, early bilateral carotid body denervation improved survival in rats with induced ischemic cardiomyopathy ${ }^{3}$.

In humans with HFrEF, stimulation of PCh with hypoxia increases sympathetic tone-measured directly with muscle sympathetic nerve activity (MSNA) ${ }^{5}$. Oversensitivity of these structures, defined as exaggerated ventilatory response to transient PCh activation with hypoxia (hypoxic ventilatory response-HVR), is a welldefined independent factor for poor prognosis in $\mathrm{HFrEF}^{6}$. Moreover, the prevalence of PCh oversensitivity is still

${ }^{1}$ Institute of Heart Diseases, Wroclaw Medical University, Borowska 213, 50-556 Wrocław, Poland. ${ }^{2}$ Institute of Heart Diseases, University Hospital, Wrocław, Poland. ${ }^{3}$ Department of Physiology, Wroclaw Medical University, Wrocław, Poland. ${ }^{4}$ Department of Cardiology, Centre for Heart Diseases, 4th Military Hospital, Wrocław, Poland. ${ }^{\bowtie}$ email: stanislaw.tubek@umw.edu.pl 
high in a contemporary HFrEF population in spite of optimal medical treatment ${ }^{7}$. Finally, PCh surgical excision significantly reduces sympathetic activity, assessed with MSNA, in pre-selected HFrEF patients ${ }^{8}$.

Surgical, percutaneous or pharmacological deactivation of PCh appears as a promising novel treatment for sympathetically mediated diseases such as HFrEF and arterial hypertension ${ }^{8-10}$. However, therapy should be tailored according to the individual pattern of reflex abnormalities engaged in the development and progression of the disease states. It could be hypothesized that only patients with increased tonic PCh input (tonicity) will benefit from the chronic PCh deactivation. Thus, it is not clear whether acute PCh activity (triggered, HVR) could serve as a reliable predictor of the favorable response. It seems that the effects of transient inhibition, rather than, the effects of transient stimulation, would better correspond with the result of procedures targeting PCh. However, so far the simple, non-invasive screening test for the augmented PCh tone is not available ${ }^{11}$.

Hyperoxia is one of a well-identified PCh inhibitors; however, it also exerts direct vasoconstrictive effect ${ }^{12-14}$. In healthy and HFrEF subjects prolonged hyperoxic exposure increases systemic vascular resistance (SVR) ${ }^{13,15,16}$. Opposite change-drop in SVR-was described by Sinski et al. in hypertensive subjects during the first minute of hyperoxic exposure ${ }^{17}$. The influence of acute hyperoxia on hemodynamics in HFrEF has not been described so far.

To further elucidate the mechanism of SVR changes and their time-dependence we designed this study in which the hemodynamic changes were assessed during one minute long exposure to $100 \%$ hyperoxia in subjects with the presumably high tonic activity of PCh (HFrEF population). These changes were then compared to healthy individuals in whom PCh tonic activity was presumed to be low. To confirm the relationship between SVR changes and the tonicity of PCh the study protocol was performed in two patients before and after bilateral carotid body resection - the procedure itself was a part of another project ${ }^{8}$. We hypothesize that the hyperoxiainduced drop in SVR in the early phase of the exposure is a result of the inhibition of the augmented tonic activity of PCh.

\section{Results}

The study protocol consisted of two parts: (1) assessment of the effects of acute hyperoxia on hemodynamic and respiratory parameters; (2) evaluation of individual peripheral chemosensitivity to hypoxia (HVR) using transient hypoxia method.

The baseline data. Parameters from one minute of baseline preceding the hyperoxic exposure (B) were averaged. LVEF, mean arterial pressure (MAP), end-tidal carbon dioxide concentration $\left(\mathrm{ETCO}_{2}\right)$ and cardiac output (CO) were significantly higher in healthy individuals, when HVR and minute ventilation (VI) were significantly higher in HFrEF patients. There were no significant differences in body mass index (BMI), haemoglobin level, creatinine serum level, heart rate (HR), systemic vascular resistance (SVR) and blood oxygen saturation $\left(\mathrm{SpO}_{2}\right)$ between studied groups. More details can be found in Table 1.

The influence of acute hyperoxia on measured parameters. Parameters from middle $20 \mathrm{~s}$ of the exposure (H1), last $20 \mathrm{~s}$ of the exposure $(\mathrm{H} 2)$ and $20 \mathrm{~s}$ following the exposure $(\mathrm{H} 3)$ were averaged and compared with B.

In HFrEF subjects hyperoxia caused initially a decrease in SVR (H1) with subsequent normalization of the parameter during $\mathrm{H} 2$ and $\mathrm{H} 3$. $\mathrm{CO}$ did not change during $\mathrm{H} 1$ and $\mathrm{H} 2$, but was reduced in $\mathrm{H} 3$. Significant increase in $\mathrm{SpO}_{2}$ and decline in VI were found during $\mathrm{H} 1, \mathrm{H} 2$ and $\mathrm{H} 3$. $\mathrm{HR}, \mathrm{MAP}$ and $\mathrm{ETCO}_{2}$ were unchanged during studied period. More details can be found in Fig. 1. All post-hoc comparisons can be found in Table 2.

In controls hyperoxia increased SVR and decreased $\mathrm{CO}$ and $\mathrm{HR}$ during $\mathrm{H} 2$ and $\mathrm{H} 3$. Significant rise in MAP and $\mathrm{SpO}_{2}$ was found during $\mathrm{H} 1, \mathrm{H} 2$ and $\mathrm{H} 3$. VI and $\mathrm{ETCO}_{2}$ remained unchanged during whole studied period. Look at Fig. 1 and Table 2 for all post-hoc comparisons.

Differences in absolute changes from baseline in measured parameters between studied groups. SVR and MAP responses to hyperoxia differed substantially between studied populations in each time-period (Fig. 2). More profound drop in VI in HFrEF subjects was observed in $\mathrm{H} 2$ and $\mathrm{H} 3$; however, a trend towards lower VI was observed also during $\mathrm{H} 1$ (Fig. 2). Changes in $\mathrm{HR}, \mathrm{CO}, \mathrm{ETCO}_{2}$ and $\mathrm{SpO} 2$ during hyperoxic challenge did not differ between studied groups ( $\mathrm{p}=\mathrm{NS}$ for all).

The influence of bilateral carotid body excision on the response to acute hyperoxia. The changes in hemodynamic parameters in response to acute hyperoxia before and after bilateral carotid body resection are presented in Figs. 3 and 4.

Interactions between hypoxic ventilatory response and hyperoxia-induced changes. There was no relationship between individual HVR and hyperoxia-induced changes in SVR, MAP, HR, CO and VI both in HFrEF and control groups ( $\mathrm{p}=\mathrm{NS}$ for all).

\section{Discussion}

In the current paper we describe time dependent changes in hemodynamic parameters caused by acute hyperoxia in the group of HFrEF patients. The observed response differs substantially between HFrEF and healthy individuals in regards to MAP and SVR. This discrepancy is caused by hyperoxia-induced inhibition of overactive PCh in HFrEF patients, which is further supported by the restoration of the normal pattern of vasomotor response following bilateral carotid body excision. 


\begin{tabular}{|c|c|c|c|}
\hline & $\begin{array}{l}\text { HFrEF } \\
\mathbf{n}=\mathbf{3 0}\end{array}$ & $\begin{array}{l}\text { Controls } \\
\mathbf{n}=\mathbf{3 0}\end{array}$ & $p$ value \\
\hline Age [year] & $62 \pm 10$ & $61 \pm 10$ & 0.92 \\
\hline Sex [male/female] & $30 / 0$ & $30 / 0$ & \\
\hline BMI $\left[\mathrm{kg}\left(\mathrm{m}^{2}\right)^{-1}\right]$ & $27.1 \pm 4$ & $27.5 \pm 5$ & 0.88 \\
\hline LVEF (\%) & $27.4 \pm 7$ & $61.2 \pm 4$ & $<0.01$ \\
\hline NTproBNP [pg ml-1] & $2804 \pm 2339$ & - & - \\
\hline $\mathrm{Hb}[\mathrm{g} \%]$ & $14.3 \pm 1.4$ & $14.6 \pm 1$ & 0.24 \\
\hline Creatinine $\left[\mathrm{mg} \mathrm{dl}^{-1}\right]$ & $1.09 \pm 0.26$ & $0.99 \pm 0.15$ & 0.2 \\
\hline Hypertension [\%] & 43 & - & - \\
\hline Diabetes [\%] & 33 & - & - \\
\hline Peak $\mathrm{VO}_{2}\left[\mathrm{ml} \mathrm{kg}^{-1} \mathrm{~min}^{-1}\right]$ & $16.6 \pm 5.4$ & - & - \\
\hline $\operatorname{HVR}\left[1 \mathrm{~min}^{-1} \%^{-1}\right]$ & $0.6 \pm 0.4$ & $0.3 \pm 0.2$ & $<0.01$ \\
\hline \multicolumn{4}{|l|}{ Therapy [\%] } \\
\hline B-blockers & $100 \%$ & - & - \\
\hline ACEI/ARB & $100 \%$ & - & - \\
\hline Aldosteron antagonists & $90 \%$ & - & - \\
\hline Loop diuretics & $70 \%$ & - & - \\
\hline Thiazides & $70 \%$ & - & - \\
\hline \multicolumn{4}{|l|}{ Baseline parameters } \\
\hline HR [bpm] & $72 \pm 11$ & $69 \pm 11$ & 0.36 \\
\hline MAP [mmHg] & $78 \pm 8$ & $88 \pm 10$ & $<0.01$ \\
\hline SVR [dyn s cm ${ }^{-5}$ ] & $1239 \pm 380$ & $1180 \pm 317$ & 0.64 \\
\hline $\mathrm{CO}\left[1 \mathrm{~min}^{-1}\right]$ & $5.61 \pm 1.3$ & $6.3 \pm 1.2$ & 0.047 \\
\hline $\mathrm{VI}\left[1 \mathrm{~min}^{-1}\right]$ & $12.1 \pm 6.2$ & $9.3 \pm 2.8$ & $<0.01$ \\
\hline $\mathrm{SpO}_{2}[\%]$ & $95 \pm 2$ & $96 \pm 2$ & 0.06 \\
\hline $\mathrm{ETCO}_{2}[\mathrm{mmHg}]$ & $34.2 \pm 5.6$ & $36.0 \pm 3.9$ & 0.03 \\
\hline
\end{tabular}

Table 1. Subjects' demographic data and baseline parameters presented as mean \pm SD. Values are presented as mean $\pm \mathrm{SD}$. HFrEF heart failure with reduced ejection fraction, $B M I$ body mass index, LVEF left ventricle ejection fraction, $N T$ proBNP N-terminal prohormone of brain natriuretic peptide, $H b$ haemoglobin level, peak $V O 2$ peak oxygen consumption, $H V R$ individual peripheral chemosensitivity to hypoxia, $A C E I$ angiotensinconverting enzyme inhibitors, $A R B$ angiotensin receptor blockers, $H R$ heart rate, MAP mean arterial pressure, $S V R$ systemic vascular resistance, $\mathrm{CO}$ cardiac output, $V I$ minute ventilation, $\mathrm{SpO}_{2}$ oxygen saturation, $\mathrm{ETCO}_{2}$ end tidal carbon dioxide.

The hemodynamic effects of sustained normobaric hyperoxia, defined as an exposure lasting from 5 to $60 \mathrm{~min}$, are well described. According to the metanalysis of the 33 studies performed in humans, published by Smit et al., hyperoxia (1) decreased HR and CO; and (2) increased SVR and MAP in healthy subjects. In HFrEF patients same effects were found regarding CO and SVR, but HR and MAP remained unchanged ${ }^{13}$. Acute (less than a minute) hyperoxia is well known to inhibit ventilation and is used to assess PCh sensitivity (Dejours test) ${ }^{18}$. In the only study, which investigated the influence of hyperoxia on hemodynamics during the first minute of the exposure, Sinski et al. described drop in MAP and SVR in hypertensive but not in healthy subjects ${ }^{17}$. To our knowledge there are no studies describing changes of hemodynamic parameters in the first minute of hyperoxic exposure in patients with HFrEF.

From physiological point of view, at the level of microvasculature, hyperoxia alters smooth muscle cells L-type $\mathrm{Ca}^{2+}$ channels conduction, increases endothelin secretion (via angiotensine II mechanism), reduces the bioavailability of nitric oxide and leads to reactive oxygen species formation ${ }^{19}$. All these actions result in an increased tone of vascular smooth muscle cells and consequent rise in SVR. In previously cited metanalysis by Smit et al. sustained hyperoxia-induced rise in SVR was twice higher in patients with HFrEF comparing to healthy individuals, what can be explained by chronic endothelial disfunction in the former group ${ }^{13,20}$. Thus, this mechanism cannot be responsible for the drop of the SVR during acute hyperoxia observed in studied HFrEF population.

However, apart from local vascular actions, hyperoxia exerts effects on the autonomic nervous system. It is known to decrease sympathetic tone, measured directly with muscle sympathetic nerve activity (MSNA), during exposures lasting for several minutes in healthy, HFrEF (with anemia) and hypertensive subjects ${ }^{21-23}$. This phenomenon is explained by the inhibition of PCh's tonic afferent signaling into brainstem sympathetic centers, leading to the decline in efferent sympathetic activity ${ }^{1}$. Nevertheless, some inconsistencies may be found in the results of these trials. For example in the paper by Seals et al. breathing 100\% oxygen decreased MSNA in seven healthy individuals ${ }^{22}$, which was not the case in the healthy control group $(n=11)$ as described by Sinski et al. ${ }^{21}$. Described inconsistences in MSNA response to hyperoxia may be a result of small sample sizes resulting in selection bias reflecting natural variability of tonic PCh activity in the studied populations. The prevalence of augmented peripheral chemoreflex sensitivity (and probably also of tonicity) is significantly different between 

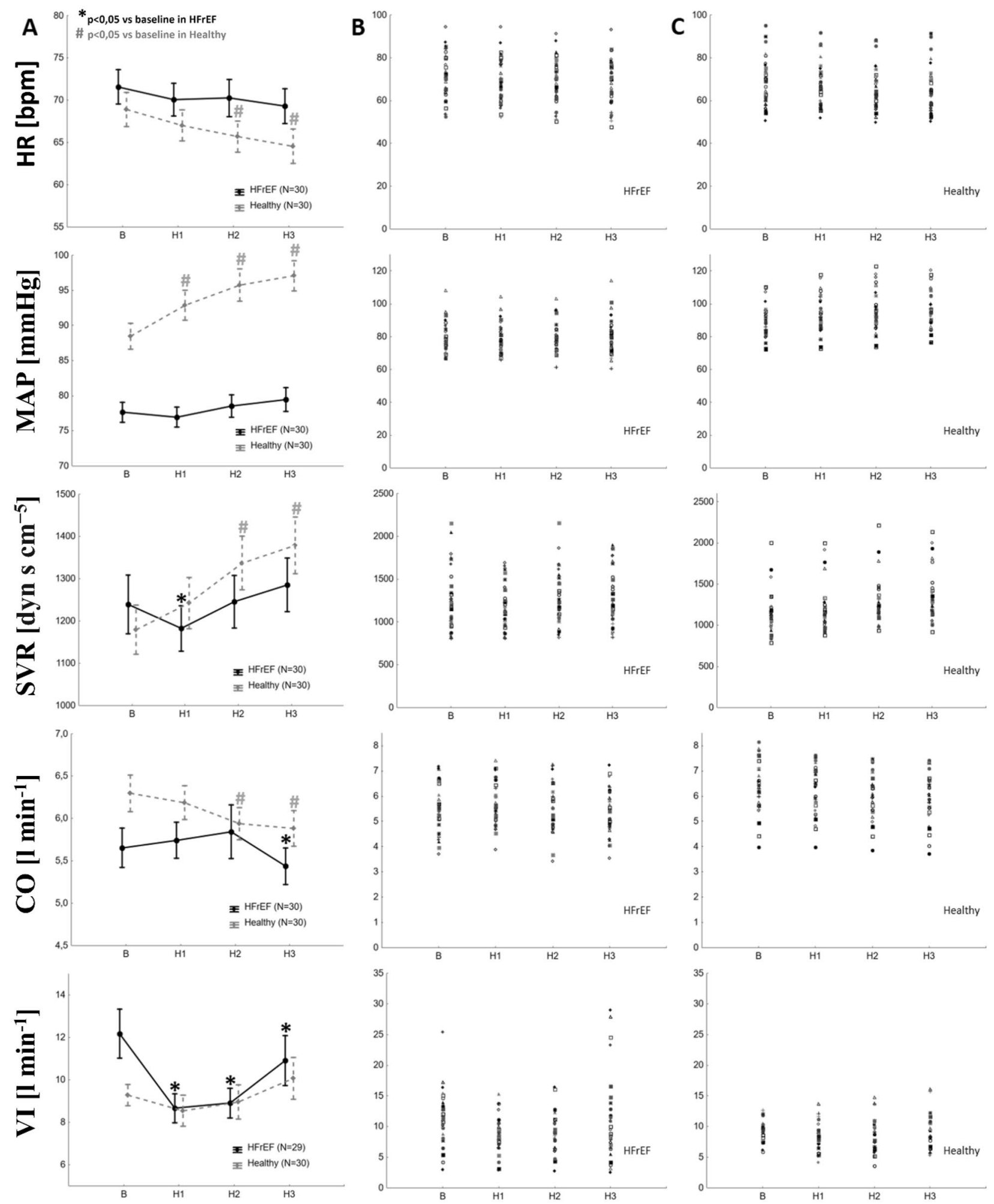

Figure 1. The influence of hyperoxia on measured parameters. Column A-changes in measured parameters (data presented as mean \pm SEM). ${ }^{*} \mathrm{p}<0.05$ vs. baseline in the post-hoc analysis for HFrEF; ${ }^{*} \mathrm{p}<0.05$ vs. baseline in the post-hoc analysis for Controls. Column B-individual data in HFrEF. Column C-individual data in Controls. 


\begin{tabular}{|c|c|c|c|c|c|c|c|c|c|c|}
\hline \multirow[b]{2}{*}{ Measured parameter } & \multicolumn{5}{|l|}{ HFrEF } & \multicolumn{5}{|l|}{ Controls } \\
\hline & B & H1 & H2 & H3 & $p$ value & B & H1 & $\mathrm{H} 2$ & H3 & $p$ value \\
\hline HR [bpm] & $72 \pm 11$ & $70 \pm 11$ & $70 \pm 12$ & $69 \pm 11$ & 0.06 & $69 \pm 11$ & $67 \pm 10$ & $66 \pm 10^{a}$ & $65 \pm 11^{\text {ab }}$ & $<0.01$ \\
\hline MAP $[\mathrm{mmHg}]$ & $78 \pm 8$ & $77 \pm 8$ & $79 \pm 9$ & $79 \pm 9^{b}$ & 0.01 & $88 \pm 10$ & $93 \pm 12^{\mathrm{a}}$ & $96 \pm 13^{\mathrm{ab}}$ & $97 \pm 12^{2 \mathrm{ab}}$ & $<0.01$ \\
\hline SVR [dyn s cm ${ }^{-5}$ ] & $1239 \pm 380$ & $1174 \pm 299^{\mathrm{a}}$ & $1246 \pm 342^{\mathrm{b}}$ & $1285 \pm 346^{\mathrm{b}}$ & $<0.01$ & $1180 \pm 317$ & $1242 \pm 332$ & $1337 \pm 346^{\mathrm{ab}}$ & $1379 \pm 367^{\mathrm{ab}}$ & $<0.01$ \\
\hline $\mathrm{CO}\left[1 \mathrm{~min}^{-1}\right]$ & $5.61 \pm 1.3$ & $5.7 \pm 1.1$ & $5.8 \pm 1.7$ & $5.4 \pm 1.2^{\mathrm{ab}}$ & $<0.01$ & $6.3 \pm 1.2$ & $6.2 \pm 1.1$ & $5.9 \pm 1^{\mathrm{ab}}$ & $5.9 \pm 1.1^{\mathrm{ab}}$ & $<0.01$ \\
\hline VI $\left[1 \mathrm{~min}^{-1}\right]$ & $12.2 \pm 6.2$ & $8.7 \pm 3.7^{\mathrm{a}}$ & $8.9 \pm 3.8^{\mathrm{a}}$ & $11 \pm 6^{a}$ & $<0.01$ & $9.3 \pm 2.8$ & $8.5 \pm 4.1$ & $9.0 \pm 4.4$ & $10.1 \pm 5.4^{\mathrm{b}}$ & $<0.01$ \\
\hline $\mathrm{SpO}_{2}[\%]$ & $95 \pm 2$ & $97 \pm 3^{a}$ & $98 \pm 2^{\mathrm{ab}}$ & $98 \pm 2^{\mathrm{ab}}$ & $<0.01$ & $96 \pm 2$ & $98 \pm 1^{\mathrm{a}}$ & $99 \pm 1^{\mathrm{ab}}$ & $99 \pm 1^{a}$ & $<0.01$ \\
\hline $\mathrm{ETCO}_{2}[\mathrm{mmHg}]$ & $34.2 \pm 5.9$ & $34.3 \pm 6.1$ & $34.0 \pm 6.3$ & $34.0 \pm 5.6$ & 0.61 & $36.0 \pm 3.9$ & $35.5 \pm 4$ & $35.2 \pm 4.9$ & $35.3 \pm 4.5$ & 0.07 \\
\hline
\end{tabular}

Table 2. The influence of hyperoxia on measured parameters. Data presented as mean $\pm \mathrm{SD}$. Values are presented as mean \pm SD. HFrEF heart failure with reduced ejection fraction, $B$ baseline, $H 1$ from 20 to $40 \mathrm{~s}$ of hyperoxia, $H 2$ last $20 \mathrm{~s}$ of the hyperoxia, $H 320 \mathrm{~s}$ following the hyperoxic exposure, $H R$ heart rate, MAP mean arterial pressure, $S V R$ systemic vascular resistance, $C O$ cardiac output, VI minute ventilation, $S_{p} \mathrm{O}_{2}$ oxygen saturation. ${ }^{\mathrm{a}} \mathrm{p}<0.05$ vs $\mathrm{B},{ }^{\mathrm{b}} \mathrm{p}<0.05$ vs $\mathrm{H} 1,{ }^{\mathrm{c}} \mathrm{p}<0.05$ vs $\mathrm{H} 2$.
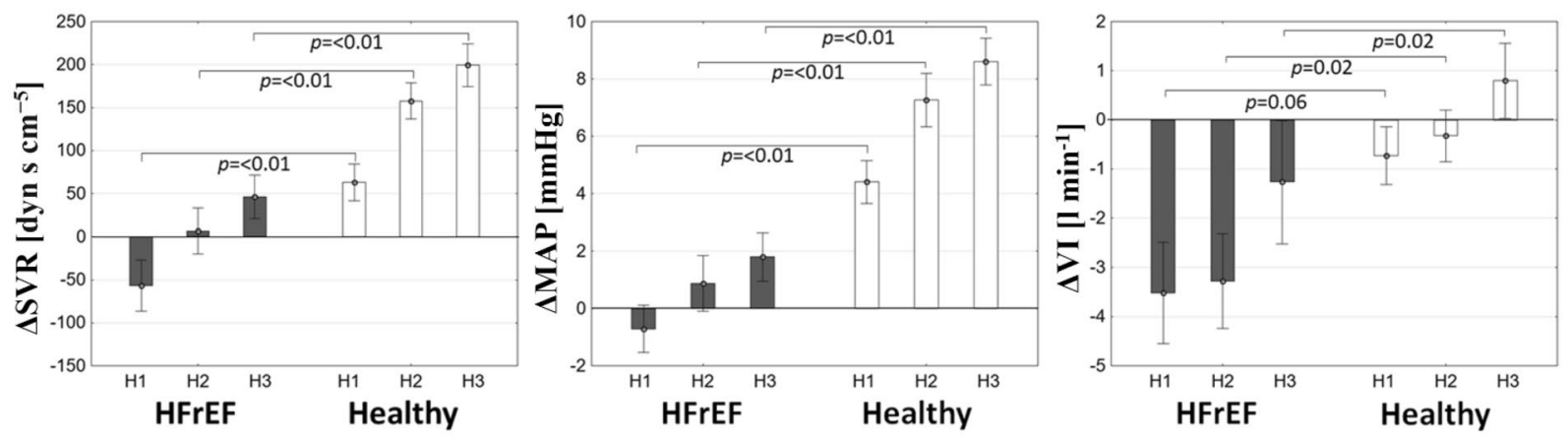

Figure 2. Differences in the response to hyperoxia between HFrEF and healthy subjects. Values of absolute change from baseline are presented as mean \pm SEM.

healthy and HFrEF subjects ( $8 \%$ and $\sim 40 \%$, respectively) $)^{11}$. The data regarding the prevalence of PCh tonicity (as measured with hyperoxia or low-dose dopamine) is missing ${ }^{11,24}$.

Both physiological mechanisms-local vasoconstriction and systemic vasodilatation-influence SVR simultaneously; however, the time-course of both responses is different. In the study by Jean-Louis et al. authors assessed changes of the retinal vessels diameter during systemic hyperoxia using fundus camera and digital image analyzer and found that arterial vasoconstriction begins $25.0 \pm 1.9 \mathrm{~s}$ after $100 \%$ oxygen administration and reaches plateau after 194.2 $\pm 12.4 \mathrm{~s}^{25}$. On the other hand direct stimulation of carotid body in vivo with adenosine caused increase in systolic blood pressure and decrease in HR with onset latency of $7.15 \pm 0.48 \mathrm{~s}$ and $4.4 \pm 0.31 \mathrm{~s}$ respectively ${ }^{26}$. Based on these data we can hypothesize that vasoconstriction (the result of local biochemical changes at the tissue level) takes more time than systemic vascular tone regulation (reflex arc via autonomic nervous system). This hypothesis may explain biphasic SVR response to hyperoxia in HFrEF patients. The primary decrease in SVR could be the result of the fast, reflex arc mediated vasodilatation due to hyperoxic inhibition of PCh. Otherwise the subsequent increase in SVR during the second phase is probably the result of delayed local vasoconstriction prevailing the primary response. The dominating role of local endothelial regulatory mechanism over autonomic regulation at microvasculature level is well known phenomenon during exercise or hypoxia ("functional sympatholysis") ${ }^{27-29}$ and according to our observations similar effect seems to be in play during sustained hyperoxia. Described model also explains monophasic SVR response to hyperoxia in healthy individuals. In these subjects PCh inhibition has no discernible effect on SVR as tonic activity of PCh is negligible. The rise in SVR is simply a result of locally driven microvascular vasoconstriction, which dominates the whole response. These observations are consistent with the results reported by Sinski et al., who also observed transient drop in SVR in hypertensive (the population with expected high prevalence of increased PCh tonicity) but not in healthy subjects following short-term hyperoxic exposure ${ }^{17}$.

In order to prove our hypothesis we included into the study two patients referred for bilateral carotid body excision and performed the study protocol before and after the procedure. Bilateral excision of carotid bodies abolished the primary (driven by the autonomic system) response to hyperoxia with no influence on the secondary, locally-mediated vasoconstriction (Figs. 3, 4).

Taking the above into consideration, acute hyperoxic exposure provides additional information regarding the influence of PCh on systemic vascular tone. The effects of PCh inhibition are masked by local vasoconstriction increasing with the time of the exposure, thus cannot be recorded during sustained hyperoxia. Among monitored parameters shift in SVR corresponds most closely with the variations in systemic vascular tone. According to our results, a significant reduction in the SVR during H1 vs baseline was found in the HFrEF 


\section{Before CBEx}
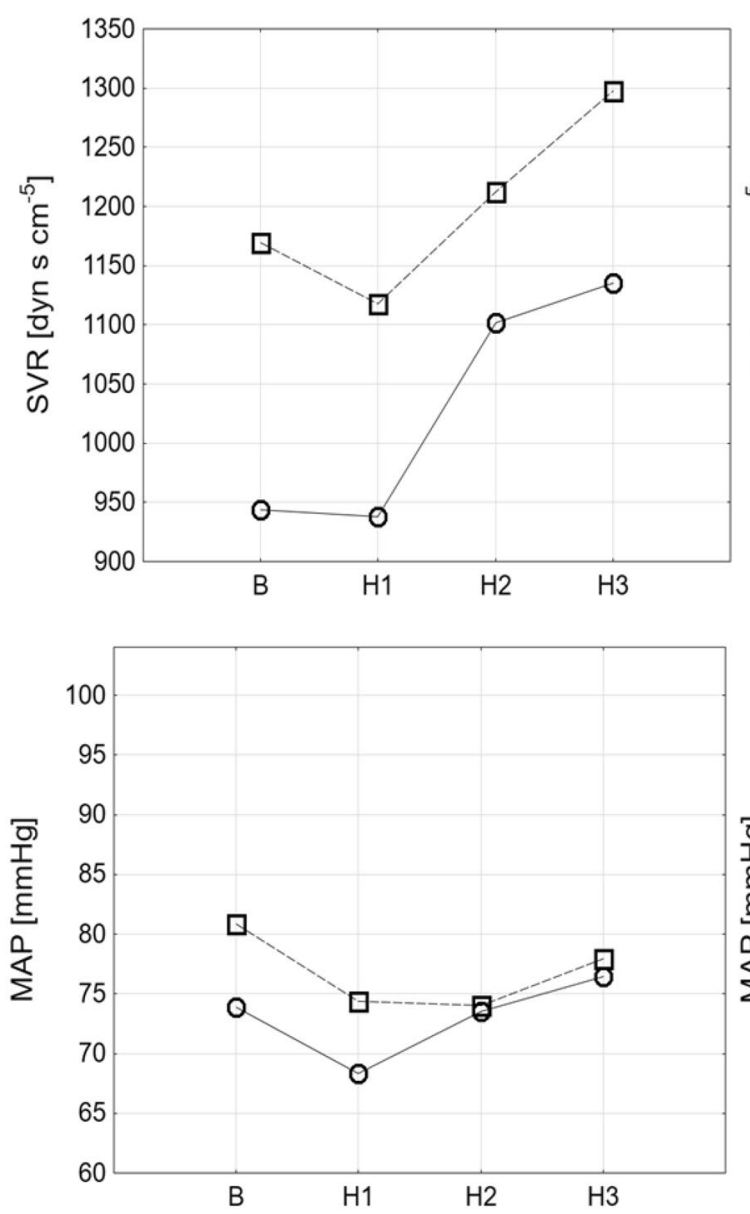

After CBEx
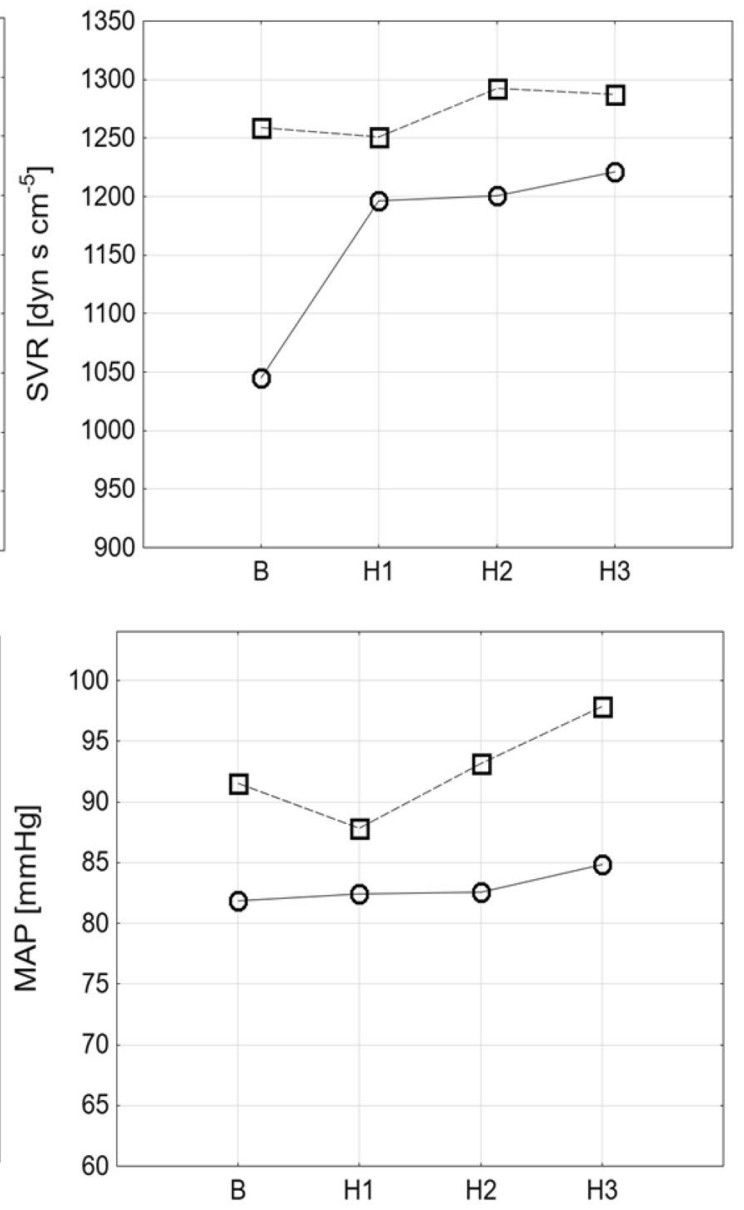

- Subject no. 1

-ษ-- Subject no. 2

Figure 3. The influence of hyperoxia on measured hemodynamic parameters following acute hyperoxia in a HFrEF patients before and after bilateral carotid bodies excision (CBEx).
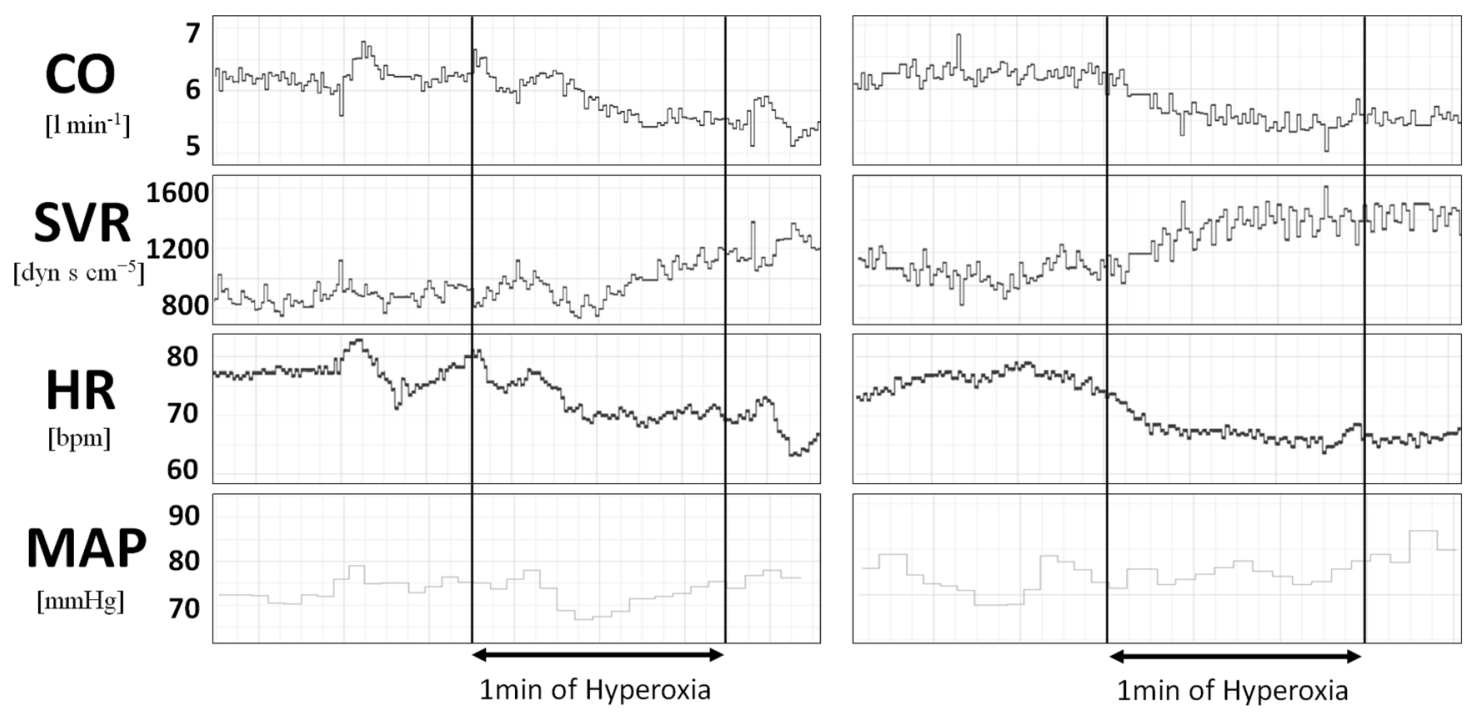

Figure 4. Hemodynamic response to hyperoxia before (left panel) and after bilateral carotid bodies excision (right panel) in HFrEF patient. 
population. Individual data shows that the prevalence of PCh tonic overactivity (defined as a drop greater than mean in healthy minus 2SD) was present in $17 \%$ of the subjects with $\mathrm{HFrEF}$ and only in $3 \%$ of healthy subjects.

In the studied group the correlation between HVR and the hyperoxia-induced changes in SVR, MAP, HR, CO and VI was not found. This may be a result of underestimation of SVR changes due to the drop of VI in HFrEF patients or of incomplete PCh inhibition by hyperoxia ${ }^{30}$. However, the lack of intuitively expected relationship between triggered reflex response and tonic activity of $\mathrm{PCh}$ is also a possibility ${ }^{31}$.

There are several limitations of the study. First of all, we did not measure sympathetic tone directly thus the influence of hyperoxia on systemic sympathetic activity remains a hypothesis. Secondly, we used non-invasive, beat-to-beat method for continuous MAP, CO and SVR monitoring, which does not include central venous pressure in SVR calculation. Nevertheless, the study aimed to assess the acute changes in measured parameters during hyperoxia, therefore the absolute values of the hemodynamic indices were not crucial for the study results. Thirdly, there were important differences in pharmacotherapy between studied groups, including drugs with vasodilatory properties (Table 1). These agents might have blunted hyperoxia-induced vasoconstriction; however, the reversal of the vascular response, from constriction to dilatation (seen as a primary response in HFrEF group) could not have been significantly influenced by underlying pharmacotherapy. Fourthly, SVR could have been affected by changes in VI, via pulmonary stretch receptors reflex. Acute hyperoxia had no influence on VI in healthy, but decreased the parameter in HFrEF patients. This might have influenced measured SVR changes, leading to underestimation of the SVR drop in HFrEF population. Fifthly, blood carbon dioxide concentration is known to influence both HVR and SVR. Baseline $\mathrm{ETCO}_{2}$ was significantly lower in HFrEF subjects, but no significant change in the parameter was found during the study period in both groups. This may again have led to an underestimation of SVR and HVR values in the HFrEF population but should not influence SVR dynamics. Finally, the study was not placebo controlled. However, patients were unaware of the timing of hyperoxic exposure due to soundless switching between inspiratory gases of the same taste and temperature.

Deactivation of the peripheral chemoreceptors in humans by interventions targeting carotid bodies (CBD) is a promising method for restoring sympathetic balance in HFrEF and hypertension. The results of the first in man studies revealed that: (1) in subjects with HFrEF and augmented HVR bilateral CBD decreased sympathetic activity and elongated exercise time $e^{8}$ and (2) in hypertensives unilateral CBD decreased blood pressure and sympathetic tone in 8 of 15 subjects with resistant arterial hypertension ${ }^{9}$. Precise subjects selection is necessary to increase the efficacy and safety of the CBD. Acute hyperoxic exposure with SVR assessment may play a role of a simple screening test; however, it should be validated in the future studies.

\section{Methods}

Studied population. The study was approved by local Ethics Committee (Komisja Bioetyczna, Wroclaw Medical University) and was performed in accordance with the latest review of the Helsinki Declaration. All subjects gave an informed written consent. Thirty HFrEF patients and 30 age- and BMI-matched healthy individuals (control group) were enrolled into the study. Additionally two patients with HFrEF referred for bilateral carotid bodies excision as a part of another project, were tested using study protocol (as described below) before and after the procedure ${ }^{8}$. All patients remained clinically stable and all HFrEF subjects received optimal medical treatment according to the current guidelines at least 1 month prior to the study entry ${ }^{32}$. Subjects excluded from study participation included those with known significant carotid artery stenosis, paced heart rhythm, suffering from significant pulmonary disease (Tiffeneau index $<70 \%$ ), left ventricular ejection fraction $<15 \%$ or $\geq 40 \%$ (for HFrEF group), NYHA functional class IV, end-stage renal failure, known history of obstructive sleep apnea and a history of an acute coronary event, coronary revascularization, or stroke within 3 months preceding the study. Study participants were asked to avoid caffeine intake $24 \mathrm{~h}$ and nicotine $12 \mathrm{~h}$ before the tests. Detailed information regarding subjects' demographic data and baseline parameters are shown in Table 1.

Assessment of the effects of acute hyperoxia. After period of familiarization with the study apparatus (of approximately $10 \mathrm{~min}$ ) when recording did not take place, subjects started breathing room air for $5 \mathrm{~min}$. Next, they were silently switched to breathing with $100 \%$ oxygen for the following $60 \mathrm{~s}$. We deliberately limited duration of the hyperoxic exposure being aware of the systemic and central actions of oxygen (including hyperoxic hyperventilation) which appear after this time ${ }^{33}$. Following the hyperoxic exposure subjects were allowed to rest until measured parameters had returned to the baseline levels. Parameters from one minute of baseline preceding the hyperoxic exposure (B), middle $20 \mathrm{~s}$ of the exposure (H1), last $20 \mathrm{~s}$ of the exposure (H2) and $20 \mathrm{~s}$ following the exposure (H3) were averaged. The parameters from the first $20 \mathrm{~s}$ of the hyperoxic survey (H0) were discarded, as during this phase oxygen concentrations in the breathing circuit and lungs were equalizing. The averaged values of the parameters recorded during B were compared with the parameters from $\mathrm{H} 1, \mathrm{H} 2$ and $\mathrm{H} 3$ respectively. We decided to use $20 \mathrm{~s}$ epochs in order to precisely characterize the time dependency of hyperoxic response.

Assessment of individual peripheral chemosensitivity to hypoxia. We employed well established method for the assessment of PCh sensitivity to intermittent hypoxia (the hypoxic ventilatory response, HVR) ${ }^{34}$. Subjects breathing room air were silently switched to $100 \%$ nitrogen for $10-35 \mathrm{~s}$, which caused falls in $\mathrm{SpO}_{2}$ to 90-65\%. Hypoxic exposures, of randomized lengths, were repeated 5-8 times per test. After each administration of nitrogen subjects were allowed to rest until measured parameters had returned to baseline levels. Each ventilatory response was calculated as an average from the three largest consecutive breaths following the nitrogen administration. HVR was expressed as the slope of the linear regression describing the relationship between the single ventilatory responses and the associated nadirs of $\mathrm{SpO}_{2}$, including the baseline values of VI and $\mathrm{SpO}_{2}$. 
Measurements. Subjects were examined in the supine position, in a quiet room, using a one-way open breathing circuit with remotely controlled mechanical valve attached to the inspiratory arm, which allowed for silent switching between room air, 100\% oxygen or $100 \%$ nitrogen. The expiratory arm was connected via a $1000 \mathrm{~L} \mathrm{~min}^{-1}$ flowhead (MLT3000L, ADInstruments) to a differential pressure transducer (FE141 Spirometer, ADInstruments) for the assessment of VI. Measured Hemodynamic parameters included: HR, CO, SVR and MAP, which were recorded non-invasively, beat-by-beat using Nexfin Finapres technique (Nexfin BMEYE B.V.). Monitor was appropriately calibrated before the test using physiological calibration. $\mathrm{SpO}_{2}$ was evaluated using a pulse oximeter (Radical-7, Masimo Corporation Irvine) with an ear clip. $\mathrm{SpO}_{2}$ recording was shifted backward by $15 \mathrm{~s}$ to compensate the circulatory delay. $\mathrm{ETCO}_{2}$ was monitored with a $\mathrm{CO}_{2}$ analyzer attached to the expiratory arm of the circuit (CapStar 100, CWE).

Data and statistical analysis. Statistica 12 (StatSoft Inc.), LabChart 8 (ADInstruments), MATLAB (MathWorks) were used to analyze the data. The distribution of the variables was tested using Shapiro-Wilk's W test. Normal distribution was found for age, haemoglobin level, baseline CO, H1 CO, H1 HR, H2 HR, H3 HR, H3 MAP; change in MAP, HR between $\mathrm{H} 1$ and baseline; change in $\mathrm{SpO}_{2}, \mathrm{MAP}$, between $\mathrm{H} 2$ and baseline; for change in MAP, $\mathrm{HR}, \mathrm{CO}, \mathrm{SpO}_{2}$ between $\mathrm{H} 3$ and baseline; all other variables were non-normally distributed. Friedman's rank test was employed to assess the effects of hyperoxia on measured parameters in subsequent epochs (B, H1, $\mathrm{H} 2$ and H3) for HFrEF and controls separately. Dunn's test was used for post-hoc analysis. Statistical testing for differences in demographic data, baseline parameters, HVR and changes in measured ventilatory and hemodynamic parameters between HFrEF and controls were performed with unpaired Student's t-test or MannWhitney U test, where appropriate. The correlations between variables were assessed with Pearson correlation coefficient or Spearman's rank correlation coefficient, where appropriate, followed by visual graph evaluation for potential non-linear relationships. Data are presented as mean and standard deviation (SD) or mean and standard error of the mean (SEM). $P$ value $<0.05$ was considered statistically significant.

Received: 20 March 2021; Accepted: 9 September 2021

Published online: 21 October 2021

\section{References}

1. Floras, J. S. \& Ponikowski, P. The sympathetic/parasympathetic imbalance in heart failure with reduced ejection fraction. Eur. Heart J. 36, 1974-1982. https://doi.org/10.1093/eurheartj/ehv087 (2015).

2. Crespo-Leiro, M. G. et al. European Society of Cardiology heart failure long-term registry (ESC-HF-LT): 1-year follow-up outcomes and differences across regions. Eur. J. Heart Fail. 18, 613-625 (2016).

3. Del Rio, R., Marcus, N. J. \& Schultz, H. D. Carotid chemoreceptor ablation improves survival in heart failure: Rescuing autonomic control of cardiorespiratory function. J. Am. Coll. Cardiol. https://doi.org/10.1016/j.jacc.2013.07.079 (2013).

4. Marcus, N. J., Rio, R., Schultz, E. P., Xia, X. H. \& Schultz, H. D. Carotid body denervation improves autonomic and cardiac function and attenuates disordered breathing in congestive heart failure. J. Physiol. 592, 391-408 (2014).

5. Di Vanna, A. et al. Blunted muscle vasodilatation during chemoreceptor stimulation in patients with heart failure. Am. J. Physiol.Heart Circ. Physiol. 293, H846-H852 (2007).

6. Ponikowski, P. et al. Peripheral chemoreceptor hypersensitivity. Circulation 104, 544-549 (2001).

7. Niewinski, P. et al. Clinical predictors and hemodynamic consequences of elevated peripheral chemosensitivity in optimally treated men with chronic systolic heart failure. J. Cardiol. Fail. 19, 408-415 (2013).

8. Niewinski, P. et al. Carotid body resection for sympathetic modulation in systolic heart failure: Results from first-in-man study. Eur. J. Heart Fail. 19, 391-400. https://doi.org/10.1002/ejhf.641 (2017).

9. Narkiewicz, K. et al. Unilateral carotid body resection in resistant hypertension: A safety and feasibility trial. JACC Basic Transl. Sci. 1, 313-324. https://doi.org/10.1016/j.jacbts.2016.06.004 (2016).

10. Pijacka, W. et al. Purinergic receptors in the carotid body as a new drug target for controlling hypertension. Nat. Med. 22, 1151-1159 (2016).

11. Tubek, S. et al. Human carotid bodies as a therapeutic target: new insights from clinician perspective. Kardiol. Polska (Polish Heart J.) 76, 1426 (2018).

12. Kumar, P. \& Prabhakar, N. R. Peripheral chemoreceptors: Function and plasticity of the carotid body. Compr. Physiol. 2, 141-219. https://doi.org/10.1002/cphy.c100069 (2012).

13. Smit, B., Smulders, Y. M., van der Wouden, J. C., Oudemans-van Straaten, H. M. \& Spoelstra-de Man, A. M. E. Hemodynamic effects of acute hyperoxia: Systematic review and meta-analysis. Crit. Care 22, 45. https://doi.org/10.1186/s13054-018-1968-2 (2018).

14. Smit, B. et al. Effects of hyperoxia on vascular tone in animal models: Systematic review and meta-analysis. Crit. Care 22, 189. https://doi.org/10.1186/s13054-018-2123-9 (2018).

15. Daly, W. J. \& Bondurant, S. Effects of oxygen breathing on the heart rate, blood pressure, and cardiac index of normal men-Resting, with reactive hyperemia, and after atropine. J. Clin. Investig. 41, 126-132 (1962).

16. Haque, W. A. et al. Hemodynamic effects of supplemental oxygen administration in congestive heart failure. J. Am. Coll. Cardiol. 27, 353-357 (1996).

17. Sinski, M. et al. Deactivation of carotid body chemoreceptors by hyperoxia decreases blood pressure in hypertensive patients. Hypertens. Res. 37, 858-862. https://doi.org/10.1038/hr.2014.91 (2014).

18. Dejours, P. Chemoreflexes in breathing. Physiol. Rev. 42, 335-358 (1962).

19. Thomson, A. J., Drummond, G. B., Waring, W. S., Webb, D. J. \& Maxwell, S. R. Effects of short-term isocapnic hyperoxia and hypoxia on cardiovascular function. J. Appl. Physiol. 101, 809-816 (2006).

20. Giannitsi, S., Bougiakli, M., Bechlioulis, A. \& Naka, K. Endothelial dysfunction and heart failure: A review of the existing bibliography with emphasis on flow mediated dilation. JRSM Cardiovasc. Dis. 8, 2048004019843047. https://doi.org/10.1177/20480 04019843047 (2019).

21. Sinski, M. et al. Tonic activity of carotid body chemoreceptors contributes to the increased sympathetic drive in essential hypertension. Hypertens. Res. 35, 487-491. https://doi.org/10.1038/hr.2011.209 (2012).

22. Seals, D. R., Johnson, D. G. \& Fregosi, R. F. Hyperoxia lowers sympathetic activity at rest but not during exercise in humans. Am. J. Physiol.-Regul. Integr. Compar. Physiol. 260, R873-R878. https://doi.org/10.1152/ajpregu.1991.260.5.R873 (1991).

23. Franchitto, N. et al. Tonic chemoreflex activation contributes to increased sympathetic nerve activity in heart failure-related anemia. Hypertension 55, 1012-1017 (2010). 
24. Niewinski, P., Tubek, S., Banasiak, W., Paton, J. F. \& Ponikowski, P. Consequences of peripheral chemoreflex inhibition with lowdose dopamine in humans. J. Physiol. 592, 1295-1308 (2014).

25. Jean-Louis, S., Lovasik, J. V. \& Kergoat, H. L. N. Systemic hyperoxia and retinal vasomotor responses. Investig. Ophthalmol. Vis. Sci. 46, 1714-1720. https://doi.org/10.1167/iovs.04-1216 (2005).

26. Tubek, S. et al. Effects of selective carotid body stimulation with adenosine in conscious humans. J. Physiol. 594, 6225-6240. https:// doi.org/10.1113/jp272109 (2016).

27. Tschakovsky, M. E., Sujirattanawimol, K., Ruble, S. B., Valic, Z. \& Joyner, M. J. Is sympathetic neural vasoconstriction blunted in the vascular bed of exercising human muscle?. J. Physiol. 541, 623-635. https://doi.org/10.1113/jphysiol.2001.014431 (2002).

28. Buckwalter, J. B., Naik, J. S., Valic, Z. \& Clifford, P. S. Exercise attenuates $\alpha$-adrenergic-receptor responsiveness in skeletal muscle vasculature. J. Appl. Physiol. 90, 172-178 (2001).

29. Dinenno, F. A. Skeletal muscle vasodilation during systemic hypoxia in humans. J. Appl. Physiol. 120, 216-225. https://doi.org/10. 1152/japplphysiol.00256.2015 (2016).

30. Duffin, J. Measuring the respiratory chemoreflexes in humans. Respir. Physiol. Neurobiol. 177, 71-79. https://doi.org/10.1016/j. resp.2011.04.009 (2011).

31. Prasad, B. et al. The need for specificity in quantifying neurocirculatory vs respiratory effects of eucapnic hypoxia and transient hyperoxia. J. Physiol. 598, 4803-4819. https://doi.org/10.1113/JP280515 (2020).

32. Ponikowski, P. et al. 2016 ESC Guidelines for the diagnosis and treatment of acute and chronic heart failure: The Task Force for the diagnosis and treatment of acute and chronic heart failure of the European Society of Cardiology (ESC) Developed with the special contribution of the Heart Failure Association (HFA) of the ESC. Eur. Heart J. 37, 2129-2200 (2016).

33. Dean, J. B., Mulkey, D. K., Henderson, R. A. 3rd., Potter, S. J. \& Putnam, R. W. Hyperoxia, reactive oxygen species, and hyperventilation: Oxygen sensitivity of brain stem neurons. J. Appl. Physiol. 1985(96), 784-791. https://doi.org/10.1152/japplphysiol.00892. 2003 (2004).

34. Chua, T. \& Coats, A. The reproducibility and comparability of tests of the peripheral chemoreflex: Comparing the transient hypoxic ventilatory drive test and the single-breath carbon dioxide response test in healthy subjects. Eur. J. Clin. Investig. 25, 887-892 (1995).

\section{Acknowledgements}

This research was financially supported from the subsidy no. SUB.E190.19.052 for the Department of heart diseases, Wroclaw Medical University, Poland.

\section{Author contributions}

S.T., P.N. and P.P. were responsible for conception and design of the experiments. S.T., P.N., B.P. and A.L. were responsible for collection of the data. S.T., P.N., B.P. and A.L. were responsible for analysis of the data and creation of the database. S.T., P.N., W.B. and P.P. were responsible for interpretation of the data. S.T. was responsible for drafting the article. S.T. and B.P. were responsible for figures and tables creation. P.N., W.B. and P.P. were responsible for revision of the manuscript. All authors approved the final version of the manuscript. All persons designated as authors qualify for authorship. All those who qualify for authorship are listed.

\section{Competing interests}

ST has received research support from Coridea and Cibiem and served as a consultant to Cibiem. PN has received research support from Coridea and Cibiem. BP none declared. AL none declared. WB none declared. PP has received research support from Coridea and Cibiem and served as a consultant to Cibiem.

\section{Additional information \\ Correspondence and requests for materials should be addressed to S.T.}

Reprints and permissions information is available at www.nature.com/reprints.

Publisher's note Springer Nature remains neutral with regard to jurisdictional claims in published maps and institutional affiliations.

(c) (1) Open Access This article is licensed under a Creative Commons Attribution 4.0 International License, which permits use, sharing, adaptation, distribution and reproduction in any medium or format, as long as you give appropriate credit to the original author(s) and the source, provide a link to the Creative Commons licence, and indicate if changes were made. The images or other third party material in this article are included in the article's Creative Commons licence, unless indicated otherwise in a credit line to the material. If material is not included in the article's Creative Commons licence and your intended use is not permitted by statutory regulation or exceeds the permitted use, you will need to obtain permission directly from the copyright holder. To view a copy of this licence, visit http://creativecommons.org/licenses/by/4.0/.

(C) The Author(s) 2021 